\title{
Fabrication of Human-Friendly Liquid Crystal Materials with $\alpha$-Ionone
}

\author{
Akane Kawaguchi, Ruri Aoki, Kenichiro Hayata, Mami Furukawa, \\ Masashi Fukawa and Seiichi Furumi* \\ Department of Applied Chemistry, Faculty of Science Division 1, \\ Tokyo University of Science, \\ 1-3 Kagurazaka, Shinjuku, Tokyo 162-8601, Japan \\ *furumi@rs.tus.ac.jp
}

\begin{abstract}
This report describes the preparation of lyotropic cholesteric liquid crystals (CLCs) with visible reflection by using two kinds of human-friendly materials. We adopted a cellulosic derivative as the main CLC component and $\alpha$-ionone as the additive liquid. A cellulosic derivative possessing propionyl side chains was dissolved in $\alpha$-ionone at the concentration between $66 \mathrm{wt} \%$ and $86 \mathrm{wt} \%$ for the formation of lyotropic CLCs. We confirmed that the visual appearance of lyotropic CLC phase shows iridescent feature such as visible Bragg reflection. The reflection peak wavelengths could be easily tuned not only by controlling the concentration of cellulosic derivative, but also by temperature. Moreover, it turned out that the refection wavelength is influenced by weight average molecular weights of the cellulosic derivatives used in lyotropic CLC mixtures. This report provides promising clues to fabricate environment- and human-friendly photonic devices by using cellulose and ionone.

Keywords: Cellulose, Ionone, Cholesteric liquid crystals, Lyotropic liquid crystals, Bragg reflection, Molecular weight effect
\end{abstract}

\section{Introduction}

To feel smell has been important sensation for creatures to live and survive since old times. Especially for human beings, the fragrances would relax our spirits and emotions. In ancient times, the natural perfumes extracted from plants were used as smoke for dedication to the altar of the gods. In addition, we used them as medicines to purify our body and mind. The modern industrial revolution brought about the breakthrough in perfumes. Until then, we used the natural aromatic products which made from trees, flowers, and fruits. However, the artificial fragrances became to be used as a result of the componential analysis of the structures in aromatic substances. Because the modern perfumes are prepared from petroleum products and natural materials, resulted in the high-throughput production at low-costs. Nowadays, we find perfumes in various products of shampoos, detergents, cosmetics, and foods.
In this way, the perfumes are very familiar presence for us that enriches our daily lives.

One representative artificial fragrance is ionone. Since ionone was for the first time synthesized by Tiemann and Krüger in 1893 [1], many reports have been hitherto made on the exploration of a wide variety of synthetic procedures [1-4]. Because of the smell of violet, ionone is widely used in soap, cosmetics and food fragrances such as peach, raspberry and strawberry.

On the other hand, the most abundant natural polymer on the Earth is cellulose. Hydroxypropyl cellulose (HPC) is prepared by etherifying hydroxyl groups of cellulose with propylene oxide. Such HPC is commercially available for food and pharmaceutical additives thanks to its inexpensive, environmentally-friendly features.

From viewpoint of liquid crystalline materials, HPC is well-known to form lyotropic cholesteric liquid crystal (CLC) phase in water [5]. 
Furthermore, the chemical modification of appropriate side chains of HPC enables the formation of lyotropic CLC phase even in various organic solvents [6-22].

In such CLC phase, chiral molecules self-assemble the periodic helicoidal structures consisting of nematic liquid crystal layers. One of the unique optical properties of CLCs is selective Bragg reflection, arising from the helical molecular structure. The non-polarized incident light propagating into the CLCs is divided into right- and left-handed circularly polarized light components by the molecular chirality. Circularly polarized light in the direction same to the molecular chirality of CLCs is reflected, and the other is transmitted. The maximum wavelength of Bragg reflection peak $(\lambda)$ is numerically expressed by the following equation,

$$
\lambda=n p
$$

where $p$ is the helical pitch length and $n$ is the average refractive index of CLCs. When the $\lambda$ value corresponds to the visible wavelength range between $400 \mathrm{~nm}$ and $700 \mathrm{~nm}$, such Bragg reflection can be visually confirmed as reflection colors [23].

In this context, the CLCs from cellulosic derivatives have been long-standing subject with considerable attention. As mentioned above, the lyotropic CLC mixtures of HPC dissolved in water would be simple and feasible, but they are quite vulnerable by heating conditions [5-9]. The organic solvents of acetone, $N, N$-dimethylacetamide, and acrylate monomer liquids have been used as a solvent to form stable CLC phase. However, these solvents are harmful to the human body. Therefore, we could not develop them to the practical applications in a straightforward way [12-22]. In this report, we describe the preparation of lyotropic CLC mixtures with visible reflection by utilizing environment and human benign materials of ionone and cellulose.

\section{Experimental}

\subsection{Materials}

Figure $1(a)$ shows the chemical structure of hydroxypropyl cellulose (HPC) derivative possessing propionyl side chains (HPC-Pr). In order to synthesize HPC-Pr, we used three kinds of HPC with different molecular weights (HPC1; (a)

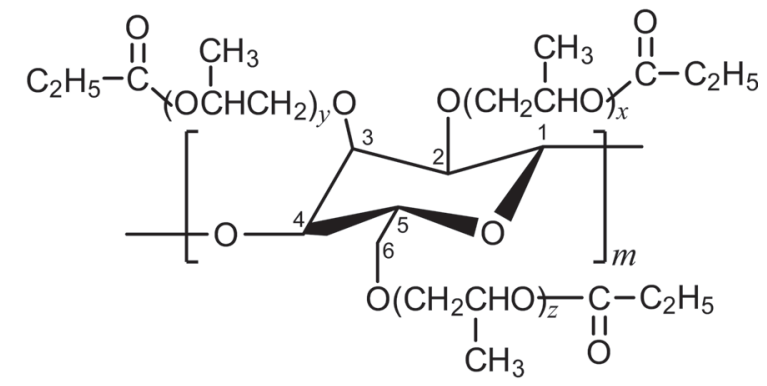

(b)

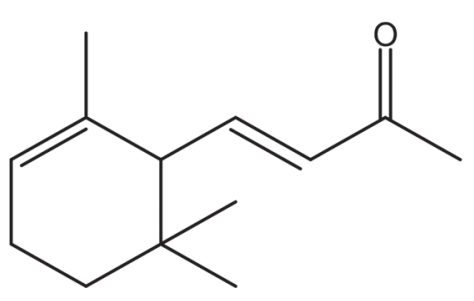

Fig. 1. Chemical structures of ( $a$ ) a hydroxypropyl cellulose (HPC) derivative possessing propionyl side chains (HPC-Pr) and (b) $\alpha$-ionone used in this study.

weight average molecular weight; $M_{w}=1.31 \times 10^{4}$, HPC $2 ; M_{w}=2.83 \times 10^{4}$, HPC $3 ; M_{w}=9.47 \times 10^{4}$ ) as the starting materials. Before the synthesis, ${ }^{1} \mathrm{H}-\mathrm{NMR}$ spectra of the pristine $\mathrm{HPCs}$ in $\mathrm{CDCl}_{3}$ were analyzed and the numbers of moles of chemically combined propylene oxide per anhydroglucose monomer unit (molar substitution; MS) in HPC1, HPC2, and HPC3 were found to be 4.03, 4.01, and 4.18, respectively. Therefore, for example HPC2, the sum of $x, y$ and $z$ shown in Fig. $1(a)$ is derived 4.01. and the average molecular weight per anhydroxyglucose monomer unit can be calculated to be $394.5 \mathrm{~g} / \mathrm{mol}$ [24].

Three kinds of HPC-Pr with different molecular weights were easily synthesized using HPC1, HPC2, or HPC3 by the esterification with propionyl chloride according to a conventional procedure in our previous reports $[22,25]$. ${ }^{1} \mathrm{H}-\mathrm{NMR}$ and FT-IR spectral analyses of products suggested the complete esterification of terminal $\mathrm{OH}$ groups of HPC by propionyl groups.

\subsection{Fabrication of CLC cells}

Figure $1(b)$ shows the chemical structure of $\alpha$-ionone. In this report, we simply referred to ionone. We adopted ionone as an organic solvent to prepare lyotropic CLC mixture at room temperature since its high boiling temperature of 
$131{ }^{\circ} \mathrm{C} / 13 \mathrm{mmHg}$. After mixing HPC-Pr in ionone for several weeks, HPC-Pr was completely dissolved in ionone. The mixture of HPC-Pr and ionone is abbreviated herein as HPC-Pr_ionone. The mixture showed Bragg reflection in the visible range at room temperature at the concentration between $66 \mathrm{wt} \%$ and $86 \mathrm{wt} \%$ of HPC-Pr.

To fabricate a CLC cell, we prepared a glass substrate covered with an alignment poly(vinyl alcohol) layer in the same way to our previous reports [22,25]. Subsequently, the mixture of HPC-Pr_ionone was placed between a pair of glass substrates. The cell gap was controlled by polytetrafluoroethylene film spacers with the thickness of $300 \mu \mathrm{m}$. After gently storing for several hours, we could obtain well-aligned CLC cells.

\section{Results and discussion}

3.1. Reflection properties of lyotropic CLC of HPC-Pr ionone mixtures

After HPC-Pr was completely dissolved in ionone, we observed lyotropic CLC phase. Particularly, HPC2-Pr_ionone mixture showed visible Bragg reflection at room temperature in the concentration range from $72 \mathrm{wt} \%$ to $78 \mathrm{wt} \%$. The transmission and reflection spectra of HPC-Pr_ionone mixtures were measured at room temperature, and the experimental results are shown in Fig. $2(a)$, and the reflection images were observed as shown in Fig. $2(b)$. In this way, the reflection wavelength and color could be widely tuned by controlling the concentration of HPC-Pr in ionone. For instance, a lyotropic CLC mixture of HPC2-Pr_ionone prepared at the HPC2-Pr concentration of $78 \mathrm{wt} \%$ exhibited violet color of Bragg reflection, whose wavelength appeared at $420 \mathrm{~nm}$, at room temperature. As polymer concentration decreased, i.e., ionone increased, the reflection peak shifted to longer wavelength. At the $\mathrm{HCP} 2-\mathrm{Pr}$ concentration of $70 \mathrm{wt} \%$ in ionone, we could not observe clear reflection color, as given in right-side image of Fig. $2(b)$. This is because the reflection peak appeared at the near infrared wavelength of $770 \mathrm{~nm}$. Such red-shifts of the reflection wavelength suggest that the CLC helical pitch length increases not only by the expansion between neighboring nematic liquid crystal layers in CLC phase by diluting with ionone, but also by the decrease in helical twisting power of HPC-Pr by ionone. (a)

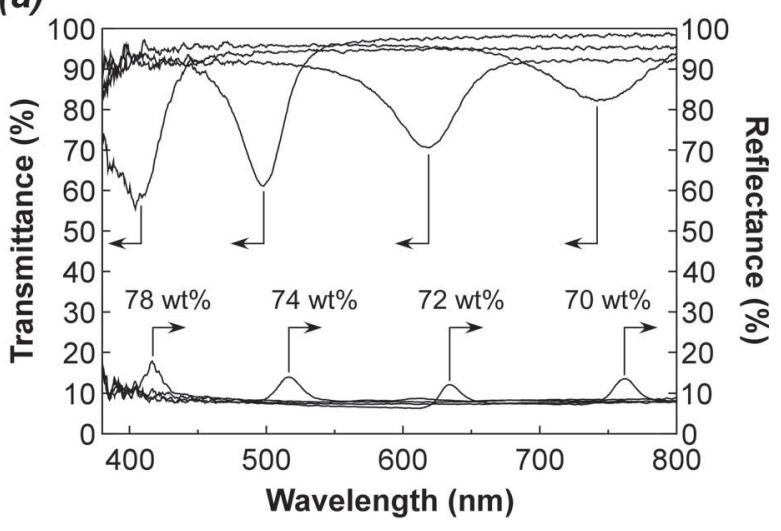

(b)

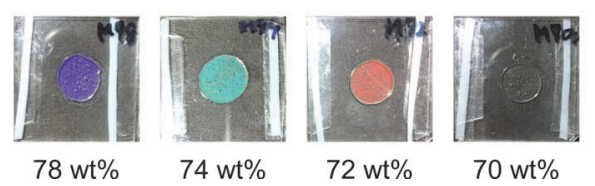

Fig. 2. (a) Changes in transmission and reflection spectra of lyotropic CLC mixtures of HPC2-Pr in ionone (HPC2-Pr_ionone) as a function of the concentration of HPC2. (b) Reflection images of the CLC cells of lyotropic CLC mixture of HPC2-Pr_ionone observed at room temperature.

\subsection{Temperature dependence of reflection peak} wavelength

When the CLC cells of HPC2-Pr ionone mixture at $78 \mathrm{wt} \%$ was heated, the reflection peak continuously shifted from $400 \mathrm{~nm}$ to longer wavelength as raising the temperature to $90{ }^{\circ} \mathrm{C}$. Figure $3(a)$ shows the changes in transmission spectrum upon heating process. Such reflection wavelength shift resulted from the extension of CLC molecular helical pitch length as elevating the temperature. Moreover, the reflection band was broadened over $65{ }^{\circ} \mathrm{C}$. This is because the molecular helical structure of lyotropic CLC deteriorates because of intensifying thermodynamic molecular motion at the relatively high temperatures. Additionally, when the CLC cell of HPC-Pr_ionone mixture at $78 \mathrm{wt} \%$ was heated over $90{ }^{\circ} \mathrm{C}$, the reflection band thoroughly disappeared by the thermally induced phase transition from CLC to isotropic phase. After cooling to room temperature from isotropic phase, the reflection band could be returned to initial wavelength of $400 \mathrm{~nm}$. We found that such reflection changes by temperature can be fully reversible.

Figure 3 (b) compiles the temperature dependence of reflection peak wavelength of HPC-Pr_ionone at the concentration range 
between $70 \mathrm{wt} \%$ and $78 \mathrm{wt} \%$. At room temperature, the reflection peak wavelengths of the mixtures were determined by the concentration of HPC-Pr in ionone. Upon heating process, each CLC cell showed the similar shift ratio of reflection peak wavelength. As mentioned in the preceding section, the twisting power of molecular helical structure increases as concentrating the HPC-Pr component in ionone. This fact leads to the stable lyotropic CLC phase up to higher temperature. In this way, we found that lyotropic CLC mixtures of HPC-Pr_ionone showed the thermotropic CLC feature in a wide temperature range.

(a)

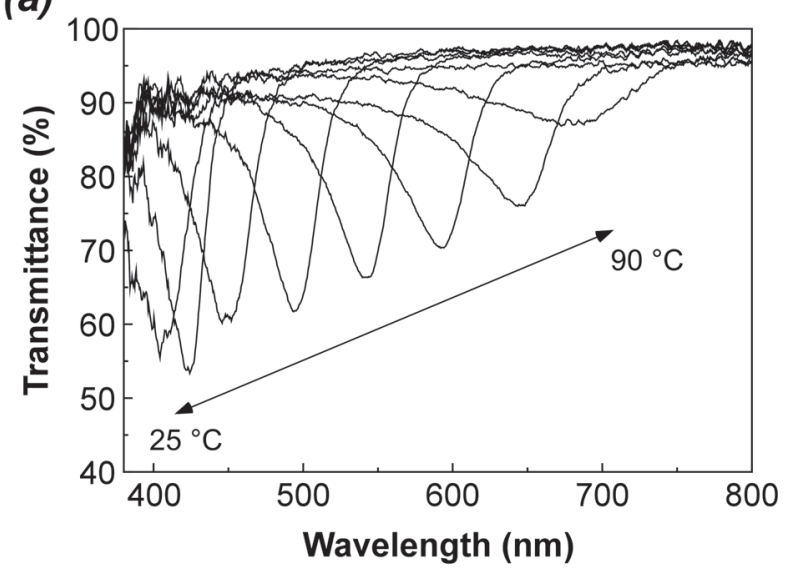

(b)

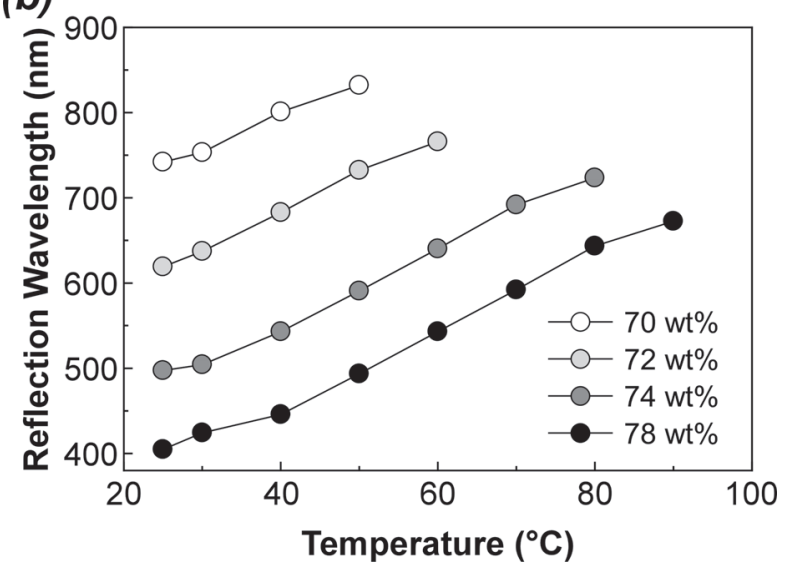

Fig. 3. (a) Transmission spectrum changes in a CLC cell of lyotropic HPC2-Pr_ionone mixtures at the concentration of $78 \mathrm{wt} \%$ upon stepwise heating from $25^{\circ} \mathrm{C}$ to $90{ }^{\circ} \mathrm{C}$. (b) Temperature dependence of the reflection peak wavelengths of lyotropic CLC cells at the various concentration range from $70 \mathrm{wt} \%$ to $78 \mathrm{wt} \%$.

3.3. Molecular weight of HPC-Pr dependence of reflection peak wavelength

In this study, three kinds of HPC-Pr were synthesized from HPC1, HPC2, and HPC3 with different molecular weights. When the HPC derivatives were dissolved in ionone, all of them exhibited the lyotropic CLC characteristic. Interestingly, we found that the concentration ranges of HPC-Pr with reflected colors are quite different. For the HPC1-Pr_ionone mixtures, visible Bragg reflection emerged at the high concentration of HPC1-Pr from 78 wt $\%$ to 86 $\mathrm{wt} \%$. In a keen contrast, the HPC3-Pr_ionone mixture showed visible Bragg reflection at the relatively low concentration from $66 \mathrm{wt} \%$ to 76 $\mathrm{wt} \%$ [26].

As mentioned above, the reflection wavelength of CLC cells of HPC-Pr_ionone mixtures changed upon stepwise heating process. This phenomenon was observed in the similar way when we changed the molecular weights of HPC-Pr. Figure 4 shows the temperature dependence of the Brag reflection peak wavelengths of HPC-Pr_ionone mixtures at the concentration of $78 \mathrm{wt} \%$. The reflection peak wavelengths of CLC cells appeared longer wavelength as the molecular weights of HPC-Pr were smaller at observed the same temperature and concentration conditions. Additionally, it was found that the thermally induced isotropic phase transition temperatures of HPC-Pr_ionone mixtures were found to be higher with increasing molecular weights of HPC-Pr. It is plausible that the molecular motion in CLC phase is restricted by increasing molecular weights of HPC-Pr, thereby resulting in the improvement of isotropic temperature. In this way, we successfully demonstrated that the reflection color of HPC-Pr_ionone mixtures are controllable

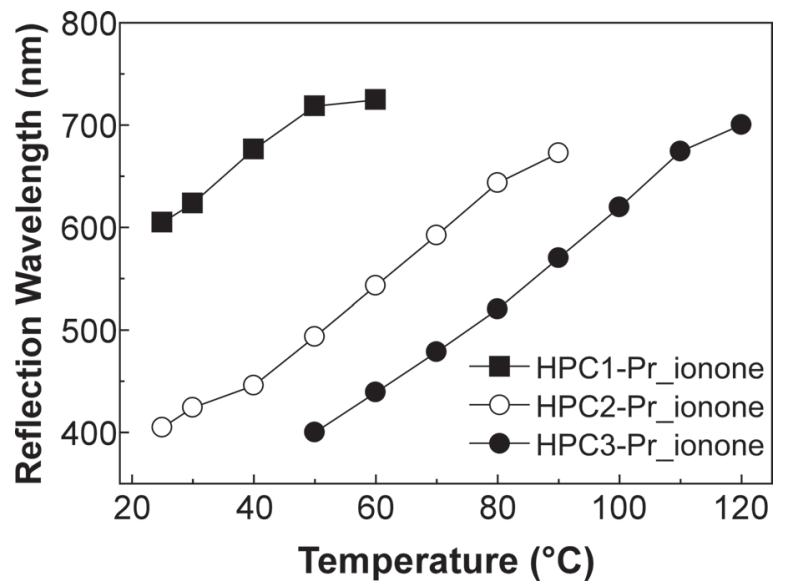

Fig. 4. Temperature dependence of the reflection peak wavelengths of CLC cells of lyotropic CLC mixtures of HPC-Pr_ionone which were prepared from HPC1-Pr (closed squares), HPC2-Pr (opened circles), and HPC3-Pr (closed circles) at the concentration of $78 \mathrm{wt} \%$. 
not only by concentration of cellulosic derivatives, but also by their molecular weights.

\section{Conclusion}

In this report, we have found that a cellulose derivative is dissolved in a liquid perfume of ionone to improve the cholesteric liquid crystallinity in a lyotropic way. The temperature dependences of Bragg reflection peak wavelengths of the mixtures could be easily controlled by changing the polymer concentration. Furthermore, the temperature dependences of reflection wavelengths were also tunable by changing the molecular weights of cellulose derivatives. This report provides useful guidelines to fabricate versatile photonic devices by utilizing environment- and human-friendly materials of cellulose as well as ionone.

\section{Acknowledgements}

One of the authors (S. F.) is deeply indebted to the Grant-in-Aid for Scientific Research (B) (No. 25288103) from the Ministry of Education, Science, Sports and Culture (MEXT) of Japan, Cosmetology Research Foundation, IMRA Japan, and Shorai Foundation for Science and Technology for financial supports on this research project.

\section{References}

1. E. Brenna, C. Fuganti, S. Serra, and P. Kraft, Eur. J. Org. Chem., (2002) 967.

2. D. Soorukram and P. Knochel, Org. Lett., 6 (2004) 2409.

3. S. Serra, C. Fuganti, and E. Brenna, Trends Biotechnol., 23 (2005), 193.

4. C. Zhang, X. Chen, N. D. Lindley, and H.-P. Too, Biotechnol. Bioeng., 115 (2018) 174.

5. R. S. Werbowyj and D. G. Gray, Mol. Cryst. Liq. Cryst., 34 (1976) 97.

6. J. S. Aspler and D. G. Gray, Macromolecules, 12 (1979) 562.

7. R. S. Werbowyj and D. G. Gray, Macromolecules, 13 (1980) 69.
8. R. S. Werbowyj and D. G. Gray, Macromolecules, 17 (1984) 1512.

9. T. Tsutusi and R. Tanaka, Polym. J., 12 (1980) 473.

10. S. M. Aharoni, Mol. Cryst. Liq. Cryst., 56 (1980) 237.

11. F. Guittard, T. Yamagishi, A. Cambon, and P. Sixou, Macromolecules, 27 (1994) 6988.

12. S.-L. Tseng, A. Vakente, and D. G. Gray, Macromolecules, 14 (1981) 715.

13. S.-L. Tseng, G. V. Laivins, and D. G. Gray, Macromolecules, 15 (1982) 1262.

14. F. Fried, J. M. Gilli, and P. Sixou, Mol. Cryst. Liq. Cryst., 98 (1983) 209.

15. C. L. McCormick, P. A. Callais, and B. H. Hutchinson, Jr., Macromolecules, 18 (1985) 2394.

16. E. Bianchi, A. Ciferri, G. Conio, and A. Tealdi, J. Polym. Sci., Part B: Polym. Phys., 27 (1989) 1477.

17. P. Ohlendorf and A. Greiner, Polym. Chem., 6 (2015) 2734.

18. M. Müller, R. Zentel, and H. Keller, $A d v$. Mater, 9 (1997) 159.

19. M. Müller and R. Zentel, Macromol. Chem. Phys., 201 (2000) 2055.

20. J. M. G. Cowie, V. Arrighi, J. Cameron, I. McEwan, and I. J. McEwen, Polymer, 42 (2001) 9657.

21. D. Wenzlik, C. Ohm, C. Serra, and R. Zentel, Soft Matter, 7 (2011) 2340.

22. M. Fukawa, K. Suzuki, and S. Furumi, J. Photopolym. Sci. Technol., 31 (2018) 563.

23. H. L. de Vries, Acta Cryst., 4 (1951) 219.

24. F. F.-L. Ho, R. R. Kohler, and G. A. Ward, Anal. Chem., 44 (1972) 178.

25. T. Ishizaki, S. Uenuma, and S. Furumi, Kobunshi Ronbunshu, 72 (2015) 737 (in Japanese).

26. P. Zugenmaier, "Handbook of Liquid Crystals”, Part IV, D. Demus, J. Goodby, G. W. Gray, H.-W. Spiess, and V. Vill, Eds., Wiley-VCH, Weinheim, 1998, p 453. 